hep-th/9512146

\title{
CANONICAL GAUGES IN THE PATH INTEGRAL FOR PARAMETRIZED SYSTEMS
}

\author{
Rafael Ferraro $^{1,2}$ and Claudio Simeone ${ }^{1}$ \\ ${ }^{1}$ Departamento de Física \\ Facultad de Ciencias Exactas y Naturales, Universidad de Buenos Aires \\ Ciudad Universitaria - Pabellón I, 1428 Buenos Aires, Argentina \\ ${ }^{2}$ Instituto de Astronomía y Física del Espacio \\ Casilla de Correo 67-Sucursal 28, 1428 Buenos Aires, Argentina
}

\begin{abstract}
:
It is well known that -differing from ordinary gauge systems- canonical gauges are not admissible in the path integral for parametrized systems. This is the case for the relativistic particle and gravitation. However, a time dependent canonical transformation can turn a parametrized system into an ordinary gauge system. It is shown how to build a canonical transformation such that the fixation of the new coordinates is equivalent to the fixation of the original ones; this aim can be achieved only if the Hamiltonian constraint allows for an intrinsic global time. Thus the resulting action, describing an ordinary gauge system and allowing for canonical gauges, can be used in the path integral for the quantum propagator associated with the original variables.
\end{abstract}




\section{Introduction}

When the transition amplitude for a gauge system is written as the sum over all histories of the exponencial of the gauge-invariant action, the path integral diverges because of the integration over the non physical degrees of freedom. This difficulty can be solved by imposing gauge conditions which select one path from each class of physically equivalent paths. Admissible gauges are those which can be reached from any path by performing gauge transformations leaving invariant the action. A gauge transformation is generated by the first class constraints $G_{a}\left(q^{i}, p_{i}\right)$,

$$
\left[G_{a}, G_{b}\right]=C_{a b}^{c}\left(q^{i}, p_{i}\right) G_{c}
$$

Under a gauge transformation the action changes by an endpoint term

$$
\delta_{\epsilon} \mathcal{S}=\left[\epsilon^{a}(\tau)\left(p_{i} \frac{\partial G_{a}}{\partial p_{i}}-G_{a}\right)\right]_{\tau_{1}}^{\tau_{2}} .
$$

If the constraints are linear and homogeneous in the momenta, as in Yang-Mills theories, the endpoint term (1.2) vanishes. If not, as it happens with generally covariant systems as the relativistic particle and gravitation, the action is invariant under a gauge transformation only if the gauge parameters $\epsilon^{a}(\tau)$ vanish at the endpoints. Thus the admissible gauges in the path integral for generally covariant systems are those which can be reached from any path by means of a gauge transformation mapping the boundaries onto themselves. This is a serious limitation to the gauge conditions to be used in the path integral: canonical gauge conditions (ie, those of the type $\chi(q, p, \tau)=0)$ cannot be used with generally covariant systems because, since the action has not gauge freedom at the boundaries, they would imply a restriction on the initial and final quantum states.

This difference was taken as the main distinction between ordinary gauge systems

and generally covariant systems ${ }^{[1]}$. However, the gauge freedom at the endpoints can be recovered if the action is modified by appropriate endpoint terms, as has been recently shown for simple systems ${ }^{[2,3]}$.

We will develop a method to obtain these terms for a generic parametrized system having only one constraint $H$, by taking them as the consequence of a time-dependent 
canonical transformation such that one of the new momenta, say $P_{0}$, coincides with the Hamiltonian constraint $H$. Then, the new variables $\left(Q^{\mu}, P_{\mu}\right), \mu \neq 0$, will be observables (although not conserved), while $Q^{0}$ will be pure gauge. In the new variables the constraint is linear and homogeneous in the momenta; thus, the action $S\left(Q^{i}, P_{i}\right)$ will have gauge freedom at the endpoints and a canonical gauge condition will be admissible. In addition the canonical transformation will be built in such a way that the quantum state $\mid q^{i}>$ is equal to $\left|Q^{i}\right\rangle$. Then the action $S\left(Q^{i}, P_{i}\right)$, which is stationary on the classical trajectory when the $Q$ 's are fixed at the boundaries, will result appropriate for computing the propagator $<q^{\prime} \mid q>$.

\section{Parametrized systems}

The action of a parametrized system reads

$$
\mathcal{S}\left[q^{i}, p_{i}, N\right]=\int_{\tau_{1}}^{\tau_{2}}\left(p_{i} \frac{d q^{i}}{d \tau}-N H\right) d \tau,
$$

where $H$ is the null Hamiltonian and the lapse function $N(\tau)$ is the Lagrange multiplier enforcing the constraint $H=0$. The constraint implies the existence of non-physical variables, which leads to an action with some kind of invariance or symmetry.

Under arbitrary changes of $q, p$ and $N$ it is obtained

$$
\delta \mathcal{S}=\left.p_{i} \delta q^{i}\right|_{\tau_{1}} ^{\tau_{2}}+\int_{\tau_{1}}^{\tau_{2}}\left[\left(\dot{q}^{i}-N \frac{\partial H}{\partial p_{i}}\right) \delta p_{i}-\left(\dot{p}_{i}+N \frac{\partial H}{\partial q^{i}}\right) \delta q^{i}-H \delta N\right] d \tau .
$$

The action is stationary on the classical path when the endpoint values of $q^{i}$ are fixed.

The action (2.1) has two different types of invariance:

1) Invariance under a reparametrization

$$
\delta q^{i}=\epsilon(\tau) \frac{d q^{i}}{d \tau}
$$




$$
\begin{gathered}
\delta p_{i}=\epsilon(\tau) \frac{d p_{i}}{d \tau}, \\
\delta N=\frac{d(N \epsilon)}{d \tau}
\end{gathered}
$$

with $\epsilon\left(\tau_{1}\right)=0=\epsilon\left(\tau_{2}\right)$. This transformation is called a reparametrization because it is equivalent to change $\tau$ by $\tau+\epsilon(\tau)$ on the path given by $q^{i}(\tau)$ and $p_{i}(\tau)$, the integral

$$
\int_{\tau_{1}}^{\tau_{2}} N(\tau) d \tau
$$

remaining unchanged. The invariance of the action (2.1) under a reparametrization means that $\tau$ is not the time but a physically irrelevant parameter. When a system is described by an action like that of (2.1), the solutions of the dynamical equations are not parametrized by $\tau$ but are

$$
q^{i}=q^{i}\left(\int^{\tau} N d \tau\right) \quad p_{i}=p_{i}\left(\int^{\tau} N d \tau\right) .
$$

So the "proper time" $\int^{\tau} N d \tau$, instead of $\tau$, plays the role of time. When the equations (2.6) can be globally solved for $\int^{\tau} N d \tau$ (ie $\int^{\tau} N d \tau=t(q, p)$ ), it is said that the system has a global phase time $t(q, p)^{[4]}$.

2) Invariance under a gauge transformation

$$
\begin{gathered}
\delta_{\epsilon} q^{i}=\epsilon(\tau)\left[q^{i}, H\right]=\epsilon(\tau) \frac{\partial H}{\partial p_{i}} \\
\delta_{\epsilon} p_{i}=\epsilon(\tau)\left[p_{i}, H\right]=-\epsilon(\tau) \frac{\partial H}{\partial q^{i}} .
\end{gathered}
$$

Then

$$
\delta_{\epsilon} \mathcal{S}=\left.p_{i} \delta_{\epsilon} q^{i}\right|_{\tau_{1}} ^{\tau_{2}}-\int_{\tau_{1}}^{\tau_{2}}\left[\epsilon(\tau)\left(\dot{q}^{i} \frac{\partial H}{\partial q^{i}}+\dot{p}_{i} \frac{\partial H}{\partial p_{i}}\right)+H \delta_{\epsilon} N\right] d \tau
$$

As $\delta_{\epsilon} N$ cannot be generated by $H$, it can be defined

$$
\delta_{\epsilon} N=\dot{\epsilon}
$$


and then

$$
\delta_{\epsilon} \mathcal{S}=\left.p_{i} \delta_{\epsilon} q^{i}\right|_{\tau_{1}} ^{\tau_{2}}-\int_{\tau_{1}}^{\tau_{2}} \frac{d}{d \tau}(\epsilon H) d \tau=\left[\epsilon(\tau)\left(p_{i} \frac{\partial H}{\partial p_{i}}-H\right)\right]_{\tau_{1}}^{\tau_{2}}
$$

On the classical path, where Hamilton equations hold, the reparametrization (2.3)-(2.5) is equivalent to a gauge transformation with parameter $N \epsilon$ and the boundary restrictions $\epsilon\left(\tau_{1}\right)=0=\epsilon\left(\tau_{2}\right)$.

If the constraint $H$ is not linear and homogeneous in the momenta, as is usual when one deals with parametrized systems, the action is not gauge-invariant unless the restrictions

$$
\epsilon\left(\tau_{1}\right)=0=\epsilon\left(\tau_{2}\right)
$$

are added.

Gauge invariance is usually regarded as the consequence of the existence of spurious degrees of freedom. However, gauge invariance of parametrized systems is related to reparametrization invariance; ie the physically irrelevant variable is not a canonical variable but is the parameter $\tau$. $\tau$ is not the time but the time can be hidden among the dynamical variables. This is the case when a global phase time exists (the Jacobi action is an example of a parametrized action which has not a global phase time ${ }^{[5]}$ ). As a result, the path integral for such a system does not depend on $\tau_{1}, \tau_{2}$, but only on the initial and final values of $q^{i}$. Hence, the path integral for a parametrized system corresponds to the probability $<q^{i^{\prime}} \mid q^{i}>$.

The restrictions (2.12) make impossible to fix the gauge in the path integral by imposing conditions on the canonical variables of the form

$$
\chi(q, p, \tau)=0
$$

("canonical gauges"). This type of gauge conditions are not admissible when the constraints are not linear and homogeneous because, due to the restrictions (2.12), there is no gauge freedom at the endpoints, and then (2.13) would imply a restriction on the initial and final quantum states ${ }^{[1]}$. 
Admissible gauges in the path integral are those which can be carried to $\chi=0$ by means of a gauge transformation leaving the action invariant. Let us consider a trajectory which differs from the condition $\chi=0$ by an infinitesimal quantity $\Delta$; the gauge transformation which makes the variables reach the gauge condition must be such that

$$
\delta_{\epsilon} \chi=-\Delta
$$

In order to have only one solution $\epsilon(\tau)$ with the boundary conditions (2.12), (2.14) should be a second order differential equation in the parameter $\epsilon$. Since $\delta_{\epsilon} N=\dot{\epsilon}$, the most obvious gauge condition could be given by a function of $\dot{N}$, namely ${ }^{[1]}$

$$
\chi=\dot{N}=0 .
$$

Any particular choice of $N(\tau)$ can be carried to $\dot{N}=0$ by successive infinitesimal gauge transformations $\delta_{\epsilon} N=\dot{\epsilon}$; these transformations are possible because there are no restrictions on $\dot{\epsilon}$, but only on $\epsilon$ at the endpoints. Gauges like (2.15) are called "derivative gauges". Although the gauge condition (2.15) does not fix the value of $N$, but only says that $N$ is constant on the trajectory, the value of $N$ is determined by the variational principle itself when the data at $\tau_{1}$ and $\tau_{2}$ are enough for knowing the global phase time $t(q, p)$ at the endpoints. In fact, $N=\Delta t / \Delta \tau$. So no ambiguities are left on the classical trajectory.

The practical value of having linear and homogeneous constraints led to distinguish these ordinary gauges systems from all others by calling them systems with internal gauge symmetries. However, there is not a true conceptual difference between both classes of systems: internal gauge symmetry can be no more than a consequence of a particular choice of variables, and an appropriate transformation $\left(q^{i}, p_{i}\right) \rightarrow\left(Q^{i}, P_{i}\right)$ can eliminate the restrictions $\epsilon\left(\tau_{1}\right)=0=\epsilon\left(\tau_{2}\right)$ on the admissible gauges, allowing us to impose canonical gauge conditions in the path integral ${ }^{[3]}$. 


\section{The endpoint terms}

As we have seen, the general form of the variation of the action under a gauge transformation is that of an endpoint term (see (2.11)). It is then possible to achieve gauge freedom at the endpoints by means of including appropriate endpoint terms in the action. These terms have been obtained in Ref. 2 for the parametrized free particle and the free relativistic particle.

In this work we develop a method that gives the appropriate endpoint terms for a parametrized system in a general way, by seeing them as a consequence of having performed a suitable canonical transformation. If the endpoint terms are called $B$, the gauge-invariant action of a parametrized system reads

$$
S\left[q^{i}, p_{i}, N\right]=\int_{\tau_{1}}^{\tau_{2}}\left(p_{i} \frac{d q^{i}}{d \tau}-N H\right) d \tau+B
$$

where, as it follows from (2.11), it is clear that it must be

$$
\delta_{\epsilon} B=\left[-\epsilon(\tau)\left(p_{i} \frac{\partial H}{\partial p_{i}}-H\right)\right]_{\tau_{1}}^{\tau_{2}}
$$

to have $\delta_{\epsilon} S=0$ for any gauge transformation.

Let us consider a complete solution $W\left(q^{i}, \alpha_{\mu}, E\right)$ of the $\tau$-independent Hamilton-Jacobi equation

$$
H\left(q^{i}, \frac{\partial W}{\partial q^{i}}\right)=E .
$$

If $E$ and the integration constants $\alpha_{\mu}$ are matched to the new momenta $\bar{P}_{0}$ and $\bar{P}_{\mu}$ respectively, then $W\left(q^{i}, \bar{P}_{j}\right)$ can be regarded as the generator function of a canonical transformation $\left(q^{i}, p_{i}\right) \rightarrow\left(\bar{Q}^{i}, \bar{P}_{i}\right)$ defined by the equations

$$
\begin{gathered}
p_{i}=\frac{\partial W}{\partial q^{i}} \\
\bar{Q}^{i}=\frac{\partial W}{\partial \bar{P}^{i}} \\
\bar{K}=N \bar{P}_{0} .
\end{gathered}
$$


As the transformation is canonical, it is clear that

$$
\begin{aligned}
& {\left[\bar{Q}^{\mu}, \bar{P}_{0}\right]=\left[\bar{Q}^{\mu}, H\right]=0} \\
& {\left[\bar{P}_{\mu}, \bar{P}_{0}\right]=\left[\bar{P}_{\mu}, H\right]=0,}
\end{aligned}
$$

which means that $\bar{Q}^{\mu}$ and $\bar{P}_{\mu}$ are (conserved) observables describing the reduced system. The dynamical evolution for $\bar{Q}^{0}$

$$
\frac{d \bar{Q}^{0}}{d \tau}=\left[\bar{Q}^{0}, \bar{K}\right]=N\left[\bar{Q}^{0}, \bar{P}_{0}\right]=N
$$

is solved by $\bar{Q}^{0}=\int^{\tau} N d \tau$. If $\bar{Q}^{0}$ is globally well defined, then $\bar{Q}^{0}$ is a global phase time. The action

$$
\bar{S}\left[\bar{Q}^{i}, \bar{P}_{i}, N\right]=\int_{\tau_{1}}^{\tau_{2}}\left(\bar{P}_{i} \frac{d \bar{Q}^{i}}{d \tau}-N \bar{P}_{0}\right) d \tau
$$

describes a parametrized system with a constraint which is linear and homogeneous in the momenta. Therefore the action $\bar{S}$ has gauge freedom at the boundaries, and does not need endpoint terms. Canonical gauges are then admissible in a path integral with the action $\bar{S}$. A canonical gauge can be chosen to be $\chi=\bar{Q}^{0}-g(\tau)$, meaning that $N(\tau)=g^{\prime}(\tau)$ on the classical trajectory.

The action $\bar{S}$ can be related with $\mathcal{S}$ by noting that

$$
p_{i} d q^{i}=d\left(W-\bar{Q}^{i} \bar{P}_{i}\right)+\bar{P}_{i} d \bar{Q}^{i}
$$

as it follows from (3.4). Then

$$
\bar{S}=\int_{\tau_{1}}^{\tau_{2}}\left(p_{i} \frac{d q^{i}}{d \tau}-N H\right) d \tau+\left[\bar{Q}^{i}\left(q^{i}, p_{i}\right) \bar{P}_{i}\left(q^{i}, p_{i}\right)-W\right]_{\tau_{1}}^{\tau_{2}}
$$

and the endpoint terms making the action $\mathcal{S}$ gauge-invariant are

$$
\bar{B}=\left[\bar{Q}^{i}\left(q^{i}, p_{i}\right) \bar{P}_{i}\left(q^{i}, p_{i}\right)-W\right]_{\tau_{1}}^{\tau_{2}}
$$

the property (3.2) is straightforwardly verified by these terms. 


\section{The variables to be fixed at the endpoints}

We have succeeded in identifying the reduced system, described by the coordinates and momenta $\left(\bar{Q}^{\mu}, \bar{P}_{\mu}\right)$, and in getting the action (3.6) which has gauge freedom at the boundaries. The added endpoint terms do not change the dynamical equations, but change the quantities to be fixed at the endpoints in order to get the trajectories from the variational principle. The action (3.6) requires fixing the $Q^{i}$ 's at the endpoints (actually only the $Q^{\mu}$ 's should be fixed, since $\bar{P}_{0}=0$ on the classical trajectory). So $\bar{S}$ is appropriate to compute $<\bar{Q}^{i^{\prime}}\left|\bar{Q}^{i}>=<\bar{Q}^{\mu \prime}, \bar{Q}^{0^{\prime}}\right| \bar{Q}^{\mu}, \bar{Q}^{0}>$-remind that $\bar{Q}^{0}$ is the global phase time- by means of a path integral allowing for canonical gauges. However, our aim is to compute $<q^{i^{\prime}} \mid q^{i}>$, instead of $<\bar{Q}^{i^{\prime}} \mid \bar{Q}^{i}>$; but $<\bar{Q}^{i^{\prime}} \mid \bar{Q}^{i}>$ is not equal to $<q^{i^{\prime}} \mid q^{i}>$, because the choice of the $\bar{Q}^{i}$ 's does not fix the same quantum state that the choice of the $q^{i}$ 's does. In fact, equations (3.4) and (3.5) tell us that the variables $\left(\bar{Q}^{\mu}, \bar{P}_{\mu}\right)$ are conserved on classical trajectories. While any classical trajectory can be characterized by the choice of the $q^{i}$ 's at the endpoints, in the new variables this is done by the choice of the conserved observables $\left(\bar{Q}^{\mu}, \bar{P}_{\mu}\right)$, and the $\bar{Q}^{0}$ 's at the endpoints. Thus, new and original variables play different roles in characterizing states or histories, and the amplitudes $<q^{i^{\prime}} \mid q^{i}>$ and $<\bar{Q}^{i^{\prime}} \mid \bar{Q}^{i}>$ have different meanings.

Still, one can look for a propagator equal to $<q^{i^{\prime}} \mid q^{i}>$ by performing a canonical transformation $\left(\bar{Q}^{\mu}, \bar{P}_{\mu}\right) \rightarrow\left(Q^{\mu}, P_{\mu}\right)$ in the reduced space. If this transformation is $\tau$-dependent the Hamiltonian will change; then the observables $Q^{\mu}$ will not be conserved, and one could succeed in getting the wished propagator. Let us consider the canonical transformation generated by

$$
F(\bar{Q}, P)=P_{0} \bar{Q}^{0}+f\left(\bar{Q}^{\mu}, P_{\mu}, \tau\right)
$$

Then

$$
\begin{gathered}
H=\bar{P}_{0}=\frac{\partial F}{\partial \bar{Q}^{0}}=P_{0} \\
\bar{Q}^{0}=\frac{\partial F}{\partial \bar{P}_{0}}=Q^{0} .
\end{gathered}
$$


The transformation $\left(\bar{Q}^{\mu}, \bar{P}_{\mu}\right) \rightarrow\left(Q^{\mu}, P_{\mu}\right)$ is generated by $f\left(\bar{Q}^{\mu}, P_{\mu}, \tau\right) . Q^{\mu}$ and $P_{\mu}$ are observables, because their Poisson brackets with $P_{0}=H$ are zero, but are not conserved because their evolution is governed by the non-zero Hamiltonian

$$
K=\bar{K}+\frac{\partial F}{\partial \tau}=N P_{0}+\frac{\partial f}{\partial \tau}
$$

( $h \equiv \partial f / \partial \tau$ is the Hamiltonian for the reduced system).

The additional endpoint term

$$
\left[Q^{i} P_{i}-F\right]_{\tau_{1}}^{\tau_{2}}=\left[Q^{\mu} P_{\mu}-f\left(\bar{Q}^{\mu}, \bar{P}_{\mu}, \tau\right)\right]_{\tau_{1}}^{\tau_{2}}
$$

depends only on observables; then it is gauge-invariant. This means that the action

$$
S\left[Q^{i}, P_{i}, N\right]=\int\left(P_{i} \frac{d Q^{i}}{d \tau}-N P_{0}-\frac{\partial f}{\partial \tau}\right) d \tau
$$

also has gauge freedom at the endpoints (which appears to be obvious if we regard that after the new canonical transformation generated by $f$ the constraint remains linear in the momenta).

The action $S\left[Q^{i}, P_{i}, N\right]$ describes a non parametrized system with internal gauge symmetry. For this system $\tau$ is the time, but $Q^{0}$ is pure gauge (of course, the roles of $\tau$ and $Q^{0}$ are interchangeable since $Q^{0}$ is a global phase time). Then, the gauge can be fixed in the path integral by means of a canonical gauge.

The action (4.4) is appropriate to compute the amplitude $<Q^{i^{\prime}}, \tau_{2} \mid Q^{i}, \tau_{1}>$

$$
<Q^{i^{\prime}}, \tau_{2}\left|Q^{i}, \tau_{1}>=\int D Q^{0} D P_{0} D Q^{\mu} D P_{\mu} D N \delta(\chi)\right|\left[\chi, P_{0}\right] \mid e^{i S},
$$

where $\chi$ is any admissible canonical gauge, and $\left|\left[\chi, P_{0}\right]\right|$ is the Fadeev-Popov determinant. Let us pay attention to the fact that this amplitude depends on $\tau_{1}$ and $\tau_{2}$, because the new action $S$ is gauge-invariant but is not invariant under reparametrizations. Of course, the path integration in eq.(4.5) is nothing but the path integral for the reduced system: the functional integration on $N$ enforces the path to lie on the constraint hypersurface, 
then by using $\chi \equiv Q^{0}-g\left(Q^{\mu}, \tau\right)$-which gives the endpoint values of $Q^{0}$ in terms of the endpoint values of $Q^{\mu}$ and $\tau-$, one integrates in $Q^{0}$ and $P_{0}$ to obtain $<Q^{\mu^{\prime}}, \tau_{2} \mid Q^{\mu}, \tau_{1}>$. In the propagator (4.5), $S$ is related to the original action by

$$
S=\int_{\tau_{1}}^{\tau_{2}}\left(p_{i} \frac{d q^{i}}{d \tau}-N H\right) d \tau+B
$$

where

$$
B \equiv\left[\bar{Q}^{i} \bar{P}_{i}-W+Q^{\mu} P_{\mu}-f\right]_{\tau_{1}}^{\tau_{2}}
$$

can be expressed as a function of the original canonical variables $q^{i}$ and $p_{i}$.

As the generator $f\left(\bar{Q}^{\mu}, P_{\mu}, \tau\right)$ has not been defined yet, one can try to define it in such a way that $<Q^{i^{\prime}}, \tau_{2} \mid Q^{i}, \tau_{1}>\left(=<Q^{\mu \prime}, \tau_{2} \mid Q^{\mu}, \tau_{1}>\right)$ coincides with $<q^{i^{\prime}} \mid q^{i}>$. In order to reach this aim one must check that

1. The constraint is such that it admits a canonical gauge $\tilde{\chi}$ (satisfying $[\tilde{\chi}, H] \not \approx 0{ }^{[6]}$ ) depending only on $\tau, q^{i}$. Then $\tilde{\chi}=0$ defines $\tau$ as a function $\tau=\tau\left(q^{i}\right)$. If so, one says that there exists an intrinsic time ${ }^{[7]}$.

If this requirement is fulfilled, one chooses the generator $f\left(\bar{Q}^{\mu}, P_{\mu}\right)$ in such a way that

2. The gauge-invariant coordinates $Q^{\mu}$ behave as coordinates on the surface $\tilde{\chi}=0$, so meaning that a particular choice of $Q^{\mu}$ and $\tau$ defines a point $q^{i}$ in the original configuration space.

In that case, $\left|Q^{\mu} \tau\right\rangle=\left|q^{i}\right\rangle$. However, the path integral (4.5) is gauge invariant; then, not only $\tilde{\chi}$ but any canonical gauge condition can be used in (4.5). So the path integral (4.5) is equal to the propagator $<q^{i^{\prime}} \mid q^{i}>$ when the generator $f\left(\bar{Q}^{\mu}, P_{\mu}\right)$ is chosen according to the prescription 2 .

A practical way to understand the prescription 2 comes of considering the eq. (4.6). In fact, while the action $S$ is stationary on the classical trajectory when the values of $Q^{\mu}$ are fixed at $\tau_{1}$ and $\tau_{2}$, the action $\mathcal{S}$ on the right hand side requires the fixation of the $q^{i}$ s. In order that both set of variables are equivalent in the gauge $\tilde{\chi}=0$, the generator $f\left(\bar{Q}^{\mu}, P_{\mu}\right)$ 
should be such that the endpoint terms vanish on the constraint hypersurface when the gauge $\tilde{\chi}=0$ is used,

$$
\left.B \equiv\left[\bar{Q}^{i} \bar{P}_{i}-W+Q^{\mu} P_{\mu}-f\right]_{\tau_{1}}^{\tau_{2}}\right|_{P_{0}=0, \tilde{\chi}=0}=0
$$

If so, the paths will be weighted by the original action $\mathcal{S}$.

In the next Section we shall apply this way of choosing the generator $f$ in several examples.

\section{Examples}

\section{5.a Parametrized free particle}

This is the system obtained when the time $t$ is included among the dynamical variables of a free particle. The parametrized particle is then described by the original variables $q$ and $p$, plus $t$ and its conjugate momentum $p_{t}$. The action of this system is

$$
\mathcal{S}\left(q, p, t, p_{t}, N\right)=\int\left(p \dot{q}+p_{t} \dot{t}-N H\right) d \tau
$$

with

$$
H=p_{t}+\frac{p^{2}}{2 m}
$$

(By solving the constraint for $p_{t}$ the action of a non relativistic particle is recovered.) A complete solution of the $\tau$-independent Hamilton-Jacobi equation is

$$
W\left(q, t, \bar{P}, \bar{P}_{0}\right)=\bar{P} q+\left(\bar{P}_{0}-\frac{\bar{P}^{2}}{2 m}\right) t .
$$

The gauge defining an intrinsic time is $\tilde{\chi} \equiv t-T(\tau)$ (for any monotonous function $T$ ) and the appropriate function $f$ making the endpoint terms vanish in this gauge is

$$
f(\bar{Q}, P, \tau)=\bar{Q} P+\frac{P^{2}}{2 m} T(\tau)
$$


The original variables $\left(q^{i}, p_{i}\right)$ are then related to the new ones by

$$
\begin{aligned}
Q^{0} & =t \\
Q & =q-\frac{P}{m}(t-T(\tau)) \\
p_{t} & =P_{0}-\frac{P^{2}}{2 m} \\
p & =P .
\end{aligned}
$$

On the constraint surface $P_{0}=0$ the endpoint terms read

$$
B=\left[-\frac{p^{2}}{2 m}(t-T(\tau))\right]_{\tau_{1}}^{\tau_{2}}
$$

and vanish in the gauge $\tilde{\chi}=0$. The amplitude $<q^{\prime} t^{\prime} \mid q t>$ can be written as

$$
\int D t D p_{t} D q D p D N \delta(\chi)|[\chi, H]| e^{i \int_{\tau_{1}}^{\tau_{2}}\left(p_{t} \frac{d t}{d \tau}+p \frac{d q}{d \tau}-N H\right) d \tau-i\left[\frac{p^{2}}{2 m}(t-T(\tau))\right]_{\tau_{1}}^{\tau_{2}}}
$$

but, since the action is gauge-invariant, the amplitude can be computed in any canonical gauge. For instance, one can choose $\chi \equiv t=0$, and obtain

$$
\begin{aligned}
<q^{\prime} t \mid q t> & =\int D q D p \quad e^{i \int_{\tau_{1}}^{\tau_{2}}\left[p \frac{d}{d \tau}\left(q+\frac{p}{m} T(\tau)\right)-\frac{p^{2}}{2 m} \frac{d T}{d \tau}\right] d \tau} \\
& =\int D Q D P e^{i \int_{\tau_{1}}^{\tau_{2}}\left(P \frac{d Q}{d \tau}-\frac{P^{2}}{2 m} \frac{d T}{d \tau}\right) d \tau}
\end{aligned}
$$

The endpoint values of $Q$ and $\tau$ are related to the endpoint values of $q$ and $t$ by the gauge condition $\tilde{\chi} \equiv t-T(\tau)=0$, in which the endpoint terms vanish:

$$
\begin{gathered}
\left.Q\right|_{\tilde{\chi}=0}=q, \\
\left.T(\tau)\right|_{\tilde{\chi}=0}=t .
\end{gathered}
$$

The path integral for the free particle is then recovered, as could be expected from the fact that the reduced system has the Hamiltonian

$$
h=\frac{P^{2}}{2 m} .
$$

Cf Ref. 2. 


\subsection{Relativistic free particle}

The Hamiltonian constraint of this system is

$$
H=\frac{1}{2 m}\left(p_{0}^{2}-p^{2}-m^{2}\right)
$$

The $\tau$-independent Hamilton-Jacobi equation has two different solutions:

$$
W_{ \pm}\left(x, x^{0}, \bar{P}, \bar{P}_{0}\right)=\bar{P} x \pm x^{0} \sqrt{\bar{P}^{2}+2 m \bar{P}_{0}+m^{2}}
$$

The gauge $\tilde{\chi} \equiv x^{0}-T(\tau)$ defines an intrinsic time. The generator $f$ making the endpoint terms vanish when $\tilde{\chi}=0$ and $P_{0}=0$ is

$$
f(\bar{Q}, P, \tau)=\bar{Q} P \mp T(\tau) \sqrt{P^{2}+m^{2}} .
$$

The relation between original and new variables is

$$
\begin{aligned}
Q^{0} & = \pm \frac{m x^{0}}{\sqrt{P^{2}+2 m P_{0}+m^{2}}} \\
Q & =x \pm \frac{P x^{0}}{\sqrt{P^{2}+2 m P_{0}+m^{2}}} \mp \frac{P T(\tau)}{\sqrt{P^{2}+m^{2}}} \\
p_{0} & = \pm \sqrt{P^{2}+2 m P_{0}+m^{2}} \\
p & =P .
\end{aligned}
$$

On the constraint surface the endpoint terms are

$$
B=\left[\mp \frac{m^{2}\left(x^{0}-T(\tau)\right)}{\sqrt{P^{2}+m^{2}}}\right]_{\tau_{1}}^{\tau_{2}}
$$

and vanish in the gauge $\tilde{\chi}=0$. The amplitude $<x^{\prime} x^{0^{\prime}} \mid x x^{0}>$ is equal to

$$
\int D x^{0} D p_{0} D x D p D N \delta(\chi)|[\chi, H]| e^{i \int_{\tau_{1}}^{\tau_{2}}\left(p_{0} \frac{d x^{0}}{d \tau}+p \frac{d x}{d \tau}-N H\right) d \tau \mp i m^{2}\left[\frac{x^{0}-T(\tau)}{\sqrt{p^{2}+m^{2}}}\right]_{\tau_{1}}^{\tau_{2}}}
$$


but it can be computed in any canonical gauge; by choosing $\chi \equiv x^{0}=0$ the following result is obtained:

$$
<x^{\prime} x^{0^{\prime}} \mid x x^{0}>=\int D x D p e^{i \int_{\tau_{1}}^{\tau_{2}} p \frac{d x}{d \tau} d \tau \pm i\left[\frac{m^{2} T(\tau)}{\sqrt{p^{2}+m^{2}}}\right]_{\tau_{1}}^{\tau_{2}}}
$$

where, with the choice $\chi \equiv x^{0}=0$,

$$
\begin{aligned}
\int_{\tau_{1}}^{\tau_{2}} p \frac{d x}{d \tau} d \tau \pm\left[\frac{m^{2} T(\tau)}{\sqrt{p^{2}+m^{2}}}\right]_{\tau_{1}}^{\tau_{2}} & =\int_{\tau_{1}}^{\tau_{2}}\left[p \frac{d}{d \tau}\left(x \mp \frac{p T(\tau)}{\sqrt{p^{2}+m^{2}}}\right) \pm \sqrt{p^{2}+m^{2}} \frac{d T}{d \tau}\right] d \tau \\
& =\int_{\tau_{1}}^{\tau_{2}}\left[P \frac{d Q}{d \tau} \pm \sqrt{P^{2}+m^{2}} \frac{d T}{d \tau}\right] d \tau
\end{aligned}
$$

The endpoint values of $Q$ and $\tau$ are related to those of $x^{0}$ and $x$ by the gauge condition such that the endpoint terms vanish:

$$
\begin{gathered}
\left.Q\right|_{\tilde{\chi}=0}=x, \\
\left.T(\tau)\right|_{\tilde{\chi}=0}=x^{0} .
\end{gathered}
$$

As it could be expected, the Hamiltonian of the reduced system is

$$
h=\mp \sqrt{P^{2}+m^{2}}
$$

and the path integral for a free relativistic particle is obtained.

\subsection{A more general constraint}

A complete solution of the Hamilton-Jacobi equation is mostly difficult to obtain. There is a simple case in two dimensions, generalizing the former examples, which can be applied to some minisuperspace models in cosmology. Let us consider a Hamiltonian constraint

$$
H\left(\phi, \Omega, \pi_{\phi}, \pi_{\Omega}\right)=g(\phi, \Omega)\left(\pi_{\phi}^{2}-\pi_{\Omega}^{2}\right)+V(\phi, \Omega)
$$


where $g(\phi, \Omega)$ and $V(\phi, \Omega)$ are positive definite functions.

Let us use null coordinates defined as

$$
u=R(\phi+\Omega), \quad v=R(\phi-\Omega)
$$

where $R$ is some monotonous function. Then

$$
\frac{1}{4}\left(\pi_{\phi}^{2}-\pi_{\Omega}^{2}\right)=R^{\prime}(\phi+\Omega) R^{\prime}(\phi-\Omega) \pi_{u} \pi_{v} .
$$

In the case that the potencial can be written as

$$
V(\phi, \Omega)=g(\phi, \Omega) L_{1}(\phi+\Omega) L_{2}(\phi-\Omega)
$$

one can factorize out a positive definite factor in $H$ by choosing $R^{\prime}$ as the positive definite function $L_{1}$ :

$$
H=4 g(\phi, \Omega) L_{2}(\phi-\Omega) L_{1}(\phi-\Omega)\left[\pi_{u} \pi_{v}+\frac{1}{4} \frac{L_{2}(\phi-\Omega)}{L_{1}(\phi-\Omega)}\right] .
$$

The function in brackets is a constraint $H^{\prime}$ equivalent to $H$, since the product of the three other factors in (5. 21) is positive definite. Therefore the canonical transformation can be generated by means of a complete solution of the $\tau$-independent Hamilton-Jacobi equation associated with

$$
H^{\prime}=\pi_{u} \pi_{v}+\frac{1}{4} L(v)
$$

where $L(v) \equiv L_{2}(\phi-\Omega) / L_{1}(\phi-\Omega)$. In such a case $P_{0}$ will not be $H$ but $H^{\prime}$. Anyway, the constraint $H$ will be linear and homogeneous in the new momentum $P_{0}$. The new variables $Q^{\mu}$ and $P_{\mu}$ will be observables, because their Poisson brackets with $H$ will be zero on the constraint surface.

The generator function $W$ is

$$
W=\bar{P} u+\frac{\bar{P}_{0}}{\bar{P}} v-\frac{1}{4 \bar{P}} \int L(v) d v .
$$

The gauge $\tilde{\chi} \equiv v-T(\tau)$ defines an intrinsic time. The generator function $f$ is

$$
f(\bar{Q}, P, \tau)=\bar{Q} P+\frac{1}{4 P} \int L(T) d T
$$


and the new variables are related to $\left\{u, \pi_{u}, v, \pi_{v}\right\}$ by

$$
\begin{aligned}
Q^{0} & =\frac{v}{P} \\
Q & =u-\frac{P_{0}}{P^{2}} v+\frac{1}{4 P^{2}} \int_{T(\tau)}^{v} L(T) d T \\
\pi_{u} & =P \\
\pi_{v} & =\frac{P_{0}}{P}-\frac{L(v)}{4 P}
\end{aligned}
$$

On the constraint surface $P_{0}=0$ the endpoint terms read

$$
B=\left[\frac{1}{2 P} \int_{T(\tau)}^{v} L(T) d T\right]_{\tau_{1}}^{\tau_{2}}
$$

and clearly vanish in the gauge $\tilde{\chi}=0$. The boundaries in the path integral are given by

$$
\begin{gathered}
\left.T\right|_{\tilde{\chi}=0}=v=R(\phi-\Omega), \\
\left.Q\right|_{\tilde{\chi}=0, P_{0}=0}=u=R(\phi+\Omega) .
\end{gathered}
$$

\section{Conclusions}

In Ref. 2 it has been signaled that a generally covariant system and an ordinary gauge system are not conceptually different. In fact, differences between both kinds of systems, which seemed to be an obstruction to the use of canonical gauges in the path integral

for generally covariant systems ${ }^{[1]}$, can be saved by improving the action principle with appropriate endpoint terms ${ }^{[2,3]}$. In this way the action is endowed with gauge freedom at the boundaries.

The improved action can be still modified by the addition of gauge invariant endpoint terms. One can take advantage of this possibility to build the endpoint terms in such a way that they cancell out on the constraint hypersurface when a gauge defining an intrinsic time $-\tau=\tau\left(q^{i}\right)$ - is used. This means that the dynamical variables to be fixed in 
the variational principle for the improved and the original action respectively, define the same physical state in both cases, so warranting that the improved action can be used in the path integral to compute the quantum propagator $<q^{i^{\prime}} \mid q^{i}>$ for the original variables. However, not all systems have an intrinsic time. For instance, the constraint of an ideal clock $[8]$

$$
H=-p^{2}+q
$$

does not admit a gauge condition $\chi(q, \tau)$ because $[\chi, H]=-p \partial \chi / \partial q$ vanishes on the constraint surface when $p=0$. In this case the solution of the $\tau$-independent Hamilton -Jacobi equation is

$$
W= \pm \frac{2}{3}\left(q-\bar{P}_{0}\right)^{3 / 2}
$$

and the global phase time $\bar{Q}^{0}$ results in

$$
\bar{Q}^{0}=\frac{\partial W}{\partial \bar{P}_{0}}= \pm \sqrt{q-\bar{P}_{0}}=p
$$

Then the gauge choice necessarily involves the momentum $p$. In this case it is said that the system has an extrinsic time ${ }^{[7]}$.

The endpoint terms can be seen as the consequence of a $\tau$-dependent canonical transformation; we have shown how this transformation can be generated in the case of a parametrized system with a constraint $H$. In this kind of systems, the constraint means that the time parameter $\tau$ has no physical meaning. The canonical transformation that generates the appropriate endpoint terms is such that $\tau$ is the time in the new system, while one of the dynamical variables $-Q^{0}$ - is pure gauge, as it happens in ordinary gauge systems.

Finally we have shown that the procedure to find the generator function $W$, leading to the identification of the reduced space, can be facilitated by appropriately scaling the constraint. 


\section{REFERENCES}

1. C. Teitelboim, Phys.Rev. D 25, 3159 (1982).

2. M. Henneaux, C. Teitelboim and J. D. Vergara, Nucl.Phys. B 387, 391 (1992).

3. M. Henneaux and C. Teitelboim, Quantization of Gauge Systems, Princeton University Press, New Jersey (1992).

4. P. Hájícek, Phys.Rev. D 34, 1040 (1986).

5. C. Lanczos, The Variational Principle of Mechanics, (Dover, New York, 1986); J. D. Brown and J. W. York, Phys.Rev. D 40, 3312 (1989).

6. P. A. M. Dirac, Lectures on Quantum Mechanics, Belfer Graduate School of Science, Yeshiva University, N.Y., 1964.

7. K. V. Kuchař, in Proceedings of the 4th Canadian Conference on General Relativity and Relativistic Astrophysics. G. Kunstatter, D. Vincent, J. Williams, World Scientific (1992).

8. S. C. Beluardi and R. Ferraro, Phys.Rev. D 52, 1963 (1995). 